\title{
Isoperimetric Polygons of Maximum Width
}

\author{
Charles Audet • Pierre Hansen · Frédéric Messine
}

Received: 17 July 2007 / Revised: 8 June 2008 / Accepted: 9 June 2008 /

Published online: 22 August 2008

(C) Springer Science+Business Media, LLC 2008

\begin{abstract}
The value $\frac{1}{2 n} \cot \left(\frac{\pi}{2 n}\right)$ is shown to be an upper bound on the width of any $n$-sided polygon with unit perimeter. This bound is reached when $n$ is not a power of 2 , and the corresponding optimal solutions are the regular polygons when $n$ is odd and clipped regular Reuleaux polygons when $n$ is even but not a power of 2 . Using a global optimization algorithm, we show that the optimal width for the quadrilateral is $\frac{1}{4} \sqrt{2(3 \sqrt{3}-3)}$ with a precision of $10^{-4}$. We propose two mathematical programs to determine the maximum width when $n=2^{s}$ with $s \geq 3$ and provide approximate, but near-optimal, solutions obtained by various heuristics and local optimization for $n=8,16$, and 32 .
\end{abstract}

Work of the first author was supported by NSERC grant 239436-01, AFOSR FA9550-07-1-0302, and ExxonMobil. Work of the second author was supported by NSERC grant 239436-01.

C. Audet $(\bowtie)$

GERAD and Département de Mathématiques et de Génie Industriel, École Polytechnique de Montréal, C.P. 6079, Succ. Centre-ville, Montréal, Québec H3C 3A7, Canada

e-mail: Charles.Audet@gerad.ca

url: www.gerad.ca/Charles.Audet

P. Hansen

GERAD and Département des Méthodes Quantitatives, HEC Montréal, 3000 Chemin de la Côte

Sainte Catherine, Montréal H3C 3A7, Canada

e-mail: Pierre.Hansen@gerad.ca

url: www.gerad.ca/Pierre.Hansen

P. Hansen

École des Hautes Études Commerciales, C.P. 6079, Succ. Centre-ville, Montréal, Québec, H3C 3A7

Canada

F. Messine

ENSEEIHT-IRIT, UMR-CNRS 5505, 2 rue Camichel BP 7122, 31071 Toulouse Cedex 7, France

e-mail: Frederic.Messine@n7.fr

url: http://www.n7.fr/ messine 
Keywords Convex polygon $\cdot$ Width $\cdot$ Perimeter $\cdot$ Reuleaux $\cdot$ Polygon

\section{Introduction}

Isoperimetric problems for polygons were first studied during the ancient Greek period. The best known example is perhaps to find which polygon with $n$ vertices and unit perimeter has maximum area. As explained in [7], this problem was solved (implicitly assuming the existence of a solution) by Zenodorus (circa 200-140 b.c.e.) in his lost treatise "On isometric figures." Fortunately, his work was reported by Pappus [23] and Theon of Alexandria [11]. The solution is the set of regular polygons. Since Reinhardt's [20] pioneering paper of 1922 on extremal properties of isodiametric and isoperimetric polygons, many other isoperimetric problems, or equivalents thereof, have been studied, see, e.g., [2, 4, 10, 12, 22, 24] and for surveys [3, 8, 9].

In the present paper, we consider another isoperimetric problem concerning the width of a polygon, i.e., the minimum distance between two parallel lines which enclose the polygon. This problem, posed in the survey [3], consists in finding for all $n$ which polygon with $n$ vertices and unit perimeter has the largest width. To the best of our knowledge, this problem is not mentioned elsewhere in the literature. A similar one [6] consists in finding polygons of maximum width and unit diameter, i.e., such that the maximum distance between two vertices is 1 . Also, [14] studies the minimum width of polytopes inscribed in convex bodies in $\mathbb{R}^{n}$, and [13] provides bounds involving the width, diameter, and perimeter of reduced convex bodies. Chapter 11 of [8] proposes related problems.

Let us call $n$-gon a polygon $\mathcal{P}_{n}$ with $n$ vertices. We use the following notation: $V\left(\mathcal{P}_{n}\right)=\left\{v_{1}, v_{2}, \ldots, v_{n}\right\}$ is the set of vertices of $\mathcal{P}_{n}$ described by their Cartesian coordinates, i.e., $v_{i}=\left(x_{i}, y_{i}\right) \in \mathbb{R}^{2}$ for $i=1,2, \ldots, n$ (indices are taken modulo $n$ ); $c_{i}$ denotes the length of the side $\left[v_{i}, v_{i+1}\right]$ of $\mathcal{P}_{n}$ and $L_{i}$ the supporting line containing that side; $p=\sum_{i=1}^{n} c_{i}$ denotes the perimeter of $\mathcal{P}_{n} ; h_{i}=\max _{x \in V} \operatorname{dist}\left(x, L_{i}\right)$ is the largest distance between a vertex $x \in V$ and the line $L_{i} ; j(i)$ is defined to be the smallest index such that $\operatorname{dist}\left(v_{j(i)}, L_{i}\right)=h_{i} ; \alpha_{i}=\angle v_{i} v_{j(i)} v_{i+1}$ is the angle between the two lines passing through $v_{i}, v_{j(i)}$ and through $v_{j(i)}, v_{i+1}$. Although the definition of $j(i)$ depends on the ordering of the points, this plays no role in the sequel. Using the above definitions, the width of the polygon is defined to be $w=\min _{i=1, \ldots, n} h_{i}=$ $\max _{h}\left\{h: h \leq h_{i}, i=1,2, \ldots, n\right\}$. The problem dealt with in this paper is of the max min max type.

In Sect. 2, we propose an upper bound on the width $w$ which will lead to the solution in almost all cases. In Sect. 3, we show that regular polygons have maximum width for all odd $n$. Moreover, clipped Reuleaux polygons [20,21] obtained by removing $n$ equal circle segments from a Reuleaux polygon are optimal when $n$ is even but not a power of 2 . So the only remaining cases are for $n=2^{s}$ with $s \geq 2$ and integer. In Sect. 4, the quadrilateral with maximum width (with a precision of $10^{-4}$ ) is determined using an interval analysis based global optimization algorithm [15-17, 19]. In Sect. 5, a nonconvex quadratic program, as well as a more concise nonconvex nonquadratic program, is proposed for finding polygons with $n=2^{s}$ vertices and extremal width. Approximate, but nonoptimal, solutions obtained by various heuritics are given for the cases where $n=8,16$, and 32 . 


\section{An Upper Bound on the Width of a Unit Perimeter Polygon with $\boldsymbol{n}$ Vertices}

Planar figures with $n$ vertices could a priori be nonconvex or nonsimple, i.e., such that at least two edges cross elsewhere than at their extremities. We next show that in such cases they are not optimal.

Proposition 1 Any planar figure with $n$ vertices $\mathcal{Q}_{n}$ and maximum width is convex and simple.

Proof Assume that $\mathcal{Q}_{n}$ is not convex, possibly nonsimple, and has maximum width. The convex hull of $\mathcal{Q}_{n}$ is a polygon $\mathcal{P}_{m}$ with $m \leq n$ vertices and with the same width as $\mathcal{Q}_{n}$ but with a strictly smaller perimeter.

Applying a homothety to $\mathcal{P}_{m}$ in order to have a perimeter equal to 1 yields a polygon with strictly larger width than that of $\mathcal{Q}_{n}$, a contradiction. Moreover, if $\mathcal{Q}_{n}$ is convex, it is simple.

We next state our main result.

Theorem 1 Let $\mathcal{P}_{n}$ be a polygon with $n \geq 3$ vertices, unit perimeter, and width $w$. Then

$$
w \leq \frac{1}{2 n} \cot \left(\frac{\pi}{2 n}\right) .
$$

Proof A simple limit argument shows that is enough to prove inequality (1) under the assumption that for each side $\left[v_{i}, v_{i+1}\right]$ of $\mathcal{P}_{n}$, there is a single vertex at a distance $h_{i}$ from $L_{i}$. This assumption allows the use of Lemma 3 of Bezdek and Fodor [6] stating that $\sum_{i=1}^{n} \alpha_{i}=\pi$. The width of the optimal polygon can be written as

$$
\begin{aligned}
& w=\max _{h, c_{i}, \alpha_{i}, h_{i}} h \\
& \text { s.t. } \quad h \leq h_{i} \quad \text { for } i=1,2, \ldots, n \\
& \\
& \quad \sum_{i=1}^{n} \alpha_{i}=\pi \\
& \\
& \quad \sum_{i=1}^{n} c_{i}=1 \\
& c_{i} \geq 0, \alpha_{i} \geq 0 \text { for } i=1,2, \ldots, n,
\end{aligned}
$$

where $h_{i}=\max _{x \in V} \operatorname{dist}\left(x, L_{i}\right)$, and $L_{i}$ is the line supporting the side $c_{i}$. Problem (2) may be relaxed by noticing that for a given angle $\alpha_{i}$ and a given side length $c_{i}$, the 
value $h_{i}$ is bounded above by $\frac{c_{i}}{2} \cot \left(\frac{\alpha_{i}}{2}\right)$ :

$$
\begin{array}{rl}
w \leq \max _{h, c_{i}, \alpha_{i}} & h \\
\text { s.t. } & h \leq \frac{c_{i}}{2} \cot \left(\frac{\alpha_{i}}{2}\right) \quad \text { for } i=1,2, \ldots, n \\
& \sum_{i=1}^{n} \alpha_{i}=\pi \\
& \sum_{i=1}^{n} c_{i}=1 \\
& c_{i} \geq 0, \alpha_{i} \geq 0 \quad \text { for } i=1,2, \ldots, n .
\end{array}
$$

Notice that $h_{i}$ does not appear in that last optimization problem. Suppose that there are indices $i$ and $j$ of an optimal solution to (3) that satisfy $0<\frac{c_{i}}{2} \cot \left(\frac{\alpha_{i}}{2}\right)<$ $\frac{c_{j}}{2} \cot \left(\frac{\alpha_{j}}{2}\right)$. Then setting

$$
c_{i}^{\prime}=\frac{\cot \left(\frac{\alpha_{j}}{2}\right)\left(c_{i}+c_{j}\right)}{\cot \left(\frac{\alpha_{i}}{2}\right)+\cot \left(\frac{\alpha_{j}}{2}\right)} \quad \text { and } \quad c_{j}^{\prime}=\frac{\cot \left(\frac{\alpha_{i}}{2}\right)\left(c_{i}+c_{j}\right)}{\cot \left(\frac{\alpha_{i}}{2}\right)+\cot \left(\frac{\alpha_{j}}{2}\right)}
$$

ensures that $c_{i}^{\prime}>0, c_{j}^{\prime}>0, c_{i}^{\prime}+c_{j}^{\prime}=c_{i}+c_{j}$ and $\frac{c_{i}^{\prime}}{2} \cot \left(\frac{\alpha_{i}}{2}\right)=\frac{c_{j}^{\prime}}{2} \cot \left(\frac{\alpha_{j}}{2}\right)>\frac{c_{i}}{2} \cot \left(\frac{\alpha_{i}}{2}\right)$. Therefore, the optimal solution of (3) is such that the values $\frac{c_{i}}{2} \cot \left(\frac{\alpha_{i}}{2}\right)$ are equal to $h$ for every index $i$. Thus

$$
\begin{array}{ll}
w \leq \max _{h, c_{i}, \alpha_{i}} & h \\
\text { s.t. } \quad & \tan \left(\frac{\alpha_{i}}{2}\right)=\frac{c_{i}}{2 h} \quad \text { for } i=1,2, \ldots, n \\
& \sum_{i=1}^{n} \alpha_{i}=\pi \\
& \sum_{i=1}^{n} c_{i}=1 \\
& c_{i} \geq 0, \alpha_{i} \geq 0 \quad \text { for } i=1,2, \ldots, n \\
\max _{h, c_{i}, \alpha_{i}} & h \\
\text { s.t. } \quad \sum_{i=1}^{n} \tan \left(\frac{\alpha_{i}}{2}\right)=\sum_{i=1}^{n} \frac{c_{i}}{2 h} \\
\quad \sum_{i=1}^{n} \alpha_{i}=\pi
\end{array}
$$




$$
\begin{array}{rl} 
& \sum_{i=1}^{n} c_{i}=1 \\
& c_{i} \geq 0, \alpha_{i} \geq 0 \quad \text { for } i=1,2, \ldots, n \\
=\max _{h, \alpha_{i}} & h \\
\text { s.t. } \quad & \sum_{i=1}^{n} \tan \left(\frac{\alpha_{i}}{2}\right)=\frac{1}{2 h} \\
& \sum_{i=1}^{n} \alpha_{i}=\pi \\
=\max _{\alpha_{i}} & \frac{\alpha_{i} \geq 0 \quad \text { for } i=1,2, \ldots, n}{2 \sum_{i=1}^{n} \tan \left(\frac{\alpha_{i}}{2}\right)} \\
\text { s.t. } \quad & \sum_{i=1}^{n} \alpha_{i}=\pi \\
& \frac{\alpha_{i} \geq 0 \quad \text { for } i=1,2, \ldots, n}{2 n} \cot \left(\frac{\pi}{2 n}\right) .
\end{array}
$$

The last equality follows from Lassak [13].

\section{The Unit-Perimeter $\boldsymbol{n}$-gon with Maximum Width for Values of $\boldsymbol{n}$ with an Odd Factor}

We first solve the problem for all odd values of $n$. This generalizes Corollary 7 of [13].

Proposition 2 Regular polygons $\mathcal{P}_{n}^{r}$ with unit perimeter and an odd number $n$ of vertices have maximum width

$$
w=\frac{1}{2 n} \cot \left(\frac{\pi}{2 n}\right)
$$

Proof The width of a unit perimeter regular $n$-gon $\mathcal{P}_{n}^{r}$, with $n$ odd, is equal to the bounds of Theorem 1 .

Let us now consider $n$-gons when $n$ is even but not a power of 2 . We need to introduce clipped Reuleaux polygons.

First, recall that Reuleaux polygons [21] are not, strictly speaking, polygons but have a polygonal basis, i.e., an odd polygon, regular or not, with the property that each vertex is at the same fixed distance $d$ from the two vertices of the opposite side. The Reuleaux polygon is obtained by replacing each side by a circle's arc with 
Fig. 1 Examples of regular Reuleaux polygons

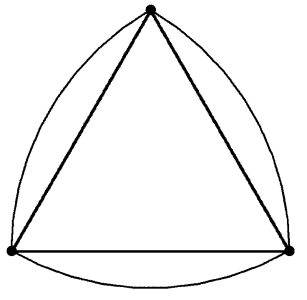

Reuleaux triangle

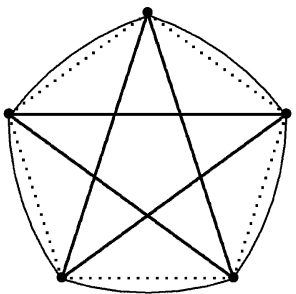

Regular Reuleaux pentagon

radius $d$, centered at a vertex and joining the two end vertices of the opposite side. Two examples of regular Reuleaux polygons are represented in Fig. 1. The diameters, also of length $d$, are represented with full lines and the sides with light ones.

Second, a clipped regular Reuleaux polygon is obtained by the following procedure:

(a) Consider a regular polygon with $m$ sides where $m$ is odd (prime or not)

(b) Transform this polygon into a Reuleaux polygon by replacing each edge by a circle's arc passing through its end vertices and centered at the opposite vertex

(c) Add at regular intervals, $2^{s}-1$ vertices within each circle's arc

(d) Take the convex hull of the vertex set, i.e., vertices of the Reuleaux polygon and vertices added in (c) or, in other words, clip $n$ equal circle segments, disjoint excepted at their vertices, from the Reuleaux polygon.

Proposition 3 Clipped regular Reuleaux polygons with unit perimeter $\mathcal{P}_{n}^{c}$ with $n$ vertices, $n=m 2^{s}$ with $m$ odd and $s$ a positive integer, have maximum width equal to

$$
w=\frac{1}{2 n} \cot \left(\frac{\pi}{2 n}\right)
$$

Proof The width of a unit perimeter clipped regular Reuleaux $n$-gon $\mathcal{P}_{n}^{c}$ with $n$ vertices, $n=m 2^{s}$ with $m$ odd and $s$ a positive integer, is equal to the bounds of Theorem 1.

Remark Proposition 2 corresponds to the particular case of Proposition 3 where $s=0$.

Examples of clipped Reuleaux polygons with maximum width and $n=6,10$, and 12 sides are given in Fig. 2. The width and diameter of the polygons also appear in the figure. Observe that if $m$ is a composite number, there are multiple optimal polygons. An example with three optimal solutions for a pentadecagon $(n=15)$ is given in Fig. 3.

Remark The unit perimeter polygons with maximum width for $n \neq 2^{s}, s \geq 2$, are the same as those with unit diameter and maximum width [6]. 


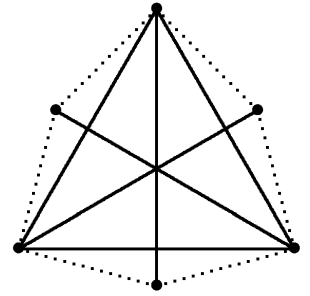

Optimal hexagon

$w_{6}=\frac{1}{12} \cot \left(\frac{\pi}{12}\right) \approx 0.3110 \ldots$

$d_{6} \approx 0.3219753 \ldots$

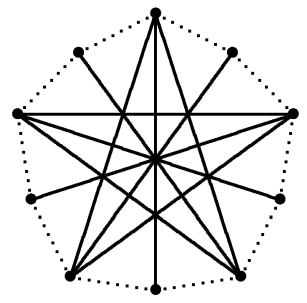

Optimal decagon

$w_{10}=\frac{1}{20} \cot \left(\frac{\pi}{20}\right) \approx 0.3157 \ldots$ $d_{10} \approx 0.3196227 \ldots$

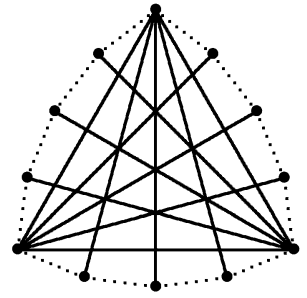

Optimal dodecagon $w_{12}=\frac{1}{24} \cot \left(\frac{\pi}{24}\right) \approx 0.3165 \ldots$ $d_{12} \approx 0.3192207 \ldots$

Fig. 2 Clipped Reuleaux polygons with maximum width, even $n$ and $n \neq 2^{s}$

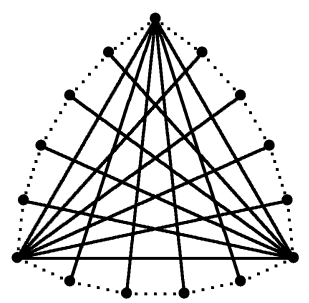

With an equilateral Reuleaux triangle

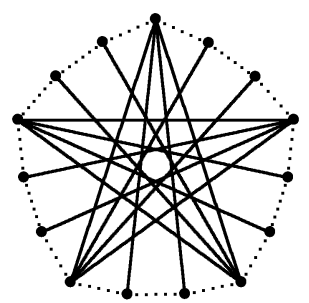

With a equilateral Reuleaux pentagon

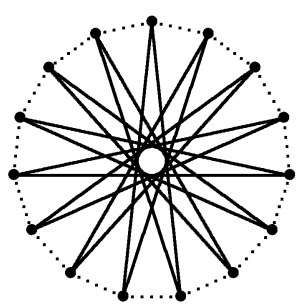

Regular pentadecagon

Fig. 3 Three pentadecagons of maximum width $w_{15}=\frac{1}{30} \cot \left(\frac{\pi}{30}\right) \approx 0.3171 \ldots$

\section{The Unit-Perimeter Quadrilateral with Maximum Width}

The remaining cases are those for which the bound of Theorem 1 might not be attained, i.e., those for which $n=2^{s}$ with $s \geq 2$ a positive integer. In this section, we analyse the first such case, the quadrilateral. This is done in two steps. We first solve analytically the subcase where this quadrilateral admits an axis of symmetry on a diagonal. Then, we show numerically that this solution is optimal without the symmetry assumption, with a precision of $10^{-4}$.

Theorem 2 The width of a quadrilateral with unit perimeter and a symmetry axis on a diagonal does not exceed $\frac{1}{4} \sqrt{3(2 \sqrt{3}-3)}$.

Proof Consider the quadrilateral represented in Fig. 4, which has a symmetry axis on the a vertical diagonal. Let $c_{1}$ and $c_{2}$ denote the lengths of the lower and upper edges, $h_{1}$ and $h_{2}$ the corresponding heights, $\alpha_{1}$ and $\alpha_{2}$ the angles between the vertical axis of symmetry and the supporting lines $L_{1}$ and $L_{2}$ of upper and lower sides, and $b$ half the length of the horizontal diagonal of the quadrilateral. Then

$$
b=c_{2} \sin \left(\alpha_{1}\right)=c_{1} \sin \left(\alpha_{2}\right) \quad \text { and } \quad c_{1}+c_{2}=\frac{1}{2}
$$


Fig. 4 Quadrilateral with an axis of symmetry on a diagonal

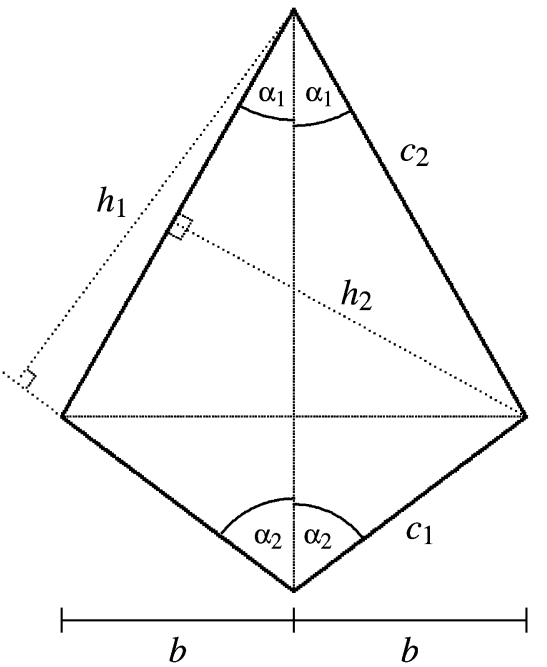

lead to

$$
c_{1}=\frac{\sin \left(\alpha_{1}\right)}{2\left(\sin \left(\alpha_{1}\right)+\sin \left(\alpha_{2}\right)\right)} \quad \text { and } \quad c_{2}=\frac{\sin \left(\alpha_{2}\right)}{2\left(\sin \left(\alpha_{1}\right)+\sin \left(\alpha_{2}\right)\right)} .
$$

Moreover,

$$
\begin{aligned}
h_{1} & =\left(c_{1} \cos \left(\alpha_{2}\right)+c_{2} \cos \left(\alpha_{1}\right)\right) \sin \left(\alpha_{2}\right) \\
& =c_{1} \sin \left(\alpha_{2}\right) \cos \left(\alpha_{2}\right)+c_{2} \cos \left(\alpha_{1}\right) \sin \left(\alpha_{2}\right) \\
& =c_{2} \sin \left(\alpha_{1}\right) \cos \left(\alpha_{2}\right)+c_{2} \cos \left(\alpha_{1}\right) \sin \left(\alpha_{2}\right)=c_{2} \sin \left(\alpha_{1}+\alpha_{2}\right) .
\end{aligned}
$$

Therefore, the question of identifying the optimal symmetrical quadrilateral may be formulated as the optimization problem

$$
\begin{array}{ll}
\max _{w, c_{2}, \alpha_{1}, \alpha_{2}} w \\
\text { s.t. } \quad w-c_{2} \sin \left(\alpha_{1}+\alpha_{2}\right) \leq 0 \\
& w-c_{2} \sin \left(2 \alpha_{1}\right) \leq 0 \\
& 2 c_{2}\left(\sin \left(\alpha_{1}\right)+\sin \left(\alpha_{2}\right)\right)-\sin \left(\alpha_{2}\right)=0 .
\end{array}
$$

Let $\mu_{1}, \mu_{2}$, and $\lambda$ be the Lagrange multipliers of the two inequality and of the equality constraints, respectively. The optimal solution must then satisfy the system of equations

$$
\begin{aligned}
& 1=\mu_{1}+\mu_{2}, \\
& 0=-\mu_{1} \sin \left(\alpha_{1}+\alpha_{2}\right)-2 \mu_{2} \sin \left(2 \alpha_{1}\right)+2 \lambda\left(\sin \left(\alpha_{1}\right)+\sin \left(\alpha_{2}\right)\right), \\
& 0=-c_{2} \mu_{1} \cos \left(\alpha_{1}+\alpha_{2}\right)-2 c_{2} \mu_{2} \cos \left(2 \alpha_{1}\right)+2 c_{2} \lambda \cos \left(\alpha_{1}\right),
\end{aligned}
$$




$$
0=-c_{2} \mu_{1} \cos \left(\alpha_{1}+\alpha_{2}\right)+2 c_{2} \lambda \cos \left(\alpha_{2}\right)-\lambda \cos \left(\alpha_{2}\right),
$$

and $\mu_{1}\left(w-h_{1}\right)=\mu_{2}\left(w-h_{2}\right)=0$. The analysis is divided into three cases.

- Case I: Suppose that $\mu_{1}=0$. Then (4) and (7) ensure that $\mu_{2}=1$ and $2 c_{2} \lambda \cos \left(\alpha_{2}\right)$ $=\lambda \cos \left(\alpha_{2}\right)$, and therefore, either $\lambda=0, \alpha_{2}=\frac{\pi}{2}$, or $c_{2}=\frac{1}{2}$. All three possibilities lead to a contradiction:

- Equation (6) and $\lambda=0$ imply that $\alpha_{1}=\frac{\pi}{4}$, which contradicts (5).

- Setting $\alpha_{2}=\frac{\pi}{2}$ yields a triangle instead of a quadrilateral, with a nonoptimal width.

- Setting $c_{2}=\frac{1}{2}$ implies that $\sin \left(\alpha_{2}\right)=\sin \left(\alpha_{1}\right)+\sin \left(\alpha_{2}\right)$ and therefore that $\alpha_{1}=0$.

Case I is therefore impossible.

- Case II: Suppose that $\mu_{2}=0$. Then (4) and (7) ensure that $\mu_{1}=1$ and $c_{2} \cos \left(\alpha_{1}+\right.$ $\left.\alpha_{2}\right)+\lambda \cos \left(\alpha_{2}\right)=2 c_{2} \lambda \cos \left(\alpha_{2}\right)$. Replacing $c_{2}$ by the value found above and substituting $\cos \left(\alpha_{1}+\alpha_{2}\right)=2 \lambda \cos \left(\alpha_{1}\right)$ (using (6)) leads to

$$
2 \lambda \sin \left(\alpha_{2}\right) \cos \left(\alpha_{1}\right)+2 \lambda\left(\sin \left(\alpha_{1}\right)+\sin \left(\alpha_{2}\right)\right) \cos \left(\alpha_{2}\right)=2 \lambda \sin \left(\alpha_{2}\right) \cos \left(\alpha_{2}\right) \text {. }
$$

This equation simplifies to

$$
\lambda \sin \left(\alpha_{2}\right) \cos \left(\alpha_{1}\right)+\lambda \sin \left(\alpha_{1}\right) \cos \left(\alpha_{2}\right)=\lambda \sin \left(\alpha_{1}+\alpha_{2}\right)=0 .
$$

It is satisfied if $\lambda=0$, which is equivalent, due to (7), to $\alpha_{1}+\alpha_{2}=\frac{\pi}{2}$. Then $h_{1}=c_{2}$, and the quadrilateral has two symmetrical right angles. Hence, $h_{2}=$ $c_{2} \sin 2 \alpha_{1}=h_{1} \sin 2 \alpha_{1} \leq h_{1}$, and the width is maximum for $\alpha_{1}=\frac{\pi}{4}=\alpha_{2}$, i.e., the square, which is not optimal. Case II is therefore settled.

- Case III: Suppose that $\mu_{1} \neq 0$ and $\mu_{2} \neq 0$. This implies that both inequality constraints must be satisfied at equality, and therefore $h_{1}=c_{2} \sin \left(\alpha_{1}+\alpha_{2}\right)$ must be equal to $h_{2}=c_{2} \sin \left(2 \alpha_{1}\right)$. It follows that either $\alpha_{2}=\alpha_{1}$ or $\alpha_{2}=\pi-3 \alpha_{1}$. We reject the solution where $\alpha_{2}=\alpha_{1}$ as it corresponds to the square. Setting $\alpha_{2}=\pi-3 \alpha_{1}$ leads to

$$
h_{1}=h_{2}=\frac{\sin \left(3 \alpha_{1}\right) \sin \left(2 \alpha_{1}\right)}{2\left(\sin \left(3 \alpha_{1}\right)+\sin \left(\alpha_{1}\right)\right)} .
$$

In order to identify the largest value that $h_{1}$ may take, we solve $h_{1}^{\prime}\left(\alpha_{1}\right)=0$. For both $\alpha_{1}$ and $\alpha_{2}$ to be nonnegative, $\alpha_{1} \in\left[\frac{\pi}{6}, \frac{\pi}{3}\right]$ must hold. Observe that $h_{1}^{\prime \prime}\left(\alpha_{1}\right)<0$ on that interval. Therefore the unique maximum is at $\alpha_{1}=\arctan (\sqrt{2 \sqrt{3}-3})$. Substituting this value of $\alpha_{1}$ in the above equations gives

$$
w=h_{1}=h_{2}=\frac{1}{4} \sqrt{3(2 \sqrt{3}-3)}, \quad c_{1}=\frac{1}{4}(3-\sqrt{3}), \quad c_{2}=\frac{1}{4}(\sqrt{3}-1) .
$$

The previous proposition gives the optimal quadrilateral under the assumption of symmetry on a diagonal. The next result shows numerically that the solution is optimal without the symmetry assumption with a precision of $10^{-4}$.

Proposition 4 The width of a quadrilateral with unit perimeter does not exceed the value $\frac{1}{4} \sqrt{3(2 \sqrt{3}-3)}+\varepsilon \approx 0.29498+\varepsilon$ with $\varepsilon<10^{-4}$. 
Fig. 5 Variables defining any quadrilateral

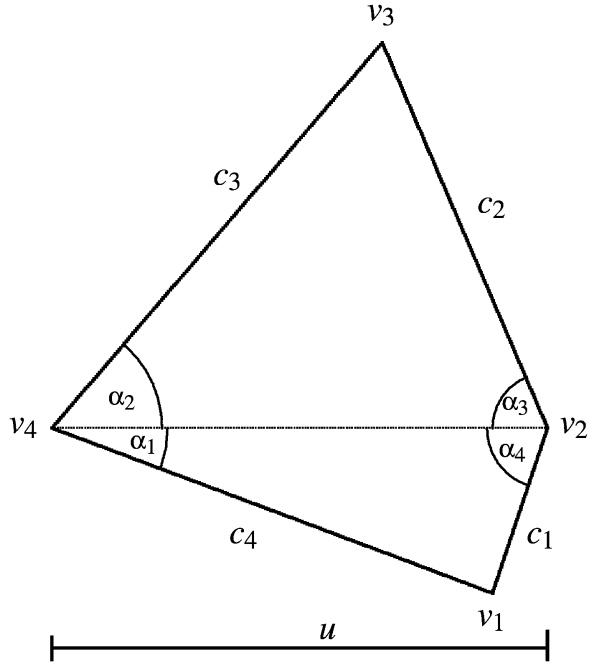

Proof Let $c_{1}, c_{2}, c_{3}$, and $c_{4}$ be the lengths of the consecutive sides of a quadrilateral, $L_{1}, L_{2}, L_{3}$, and $L_{4}$ the supporting lines of these sides, $v_{1}$ the vertex at the intersection of $L_{1}$ and $L_{4}$, and $v_{i+1}$ the vertex at the intersection of $L_{i}$ and $L_{i+1}$ for $i=1,2,3$.

By symmetry, we may assume that $v_{4}$ is not closer than $v_{3}$ to the line $L_{1}$. Consequently, the distance from $v_{2}$ cannot be closer than $v_{1}$ to the line $L_{3}$. Therefore $h_{1}=\operatorname{dist}\left(v_{4}, L_{1}\right)$ and $h_{3}=\operatorname{dist}\left(v_{1}, L_{3}\right)$.

Again, by symmetry, we may assume that $v_{4}$ is not closer than $v_{1}$ to the line $L_{2}$. Consequently, $v_{2}$ cannot be closer than $v_{3}$ to the line $L_{4}$. Therefore $h_{2}=\operatorname{dist}\left(v_{4}, L_{2}\right)$ and $h_{3}=\operatorname{dist}\left(v_{3}, L_{4}\right)$.

Now, let $u$ denote the distance from $v_{4}$ to $v_{2}$, and let $\alpha_{i}$ denote the angles as represented in Fig. 5. The above assumptions based on symmetry may be modeled as

$$
\begin{array}{ll}
h_{1}=u \sin \left(\alpha_{4}\right) \geq c_{2} \sin \left(\alpha_{3}+\alpha_{4}\right) ; & h_{2}=u \sin \left(\alpha_{3}\right) \geq c_{1} \sin \left(\alpha_{3}+\alpha_{4}\right) ; \\
h_{3}=c_{4} \sin \left(\alpha_{1}+\alpha_{2}\right) \geq u \sin \left(\alpha_{2}\right) ; & h_{4}=c_{3} \sin \left(\alpha_{1}+\alpha_{2}\right) \geq u \sin \left(\alpha_{1}\right) .
\end{array}
$$

The width of the quadrilateral is equal to $\min \left\{h_{1}, h_{2}, h_{3}, h_{4}\right\}$, or, in other words, the width is the largest scalar $w$ such that $w \leq h_{i}$ for all $i=1,2,3,4$. Adding the constraint that the perimeter is one and the constraints tying the length of the sides to the angles and to $u$, one gets the optimization problem

$$
\begin{array}{rl}
\max _{u, w, c_{i}, \alpha_{i}} & w \\
\text { s.t. } & w \leq u \sin \left(\alpha_{4}\right) \geq c_{2} \sin \left(\alpha_{3}+\alpha_{4}\right) \\
& w \leq u \sin \left(\alpha_{3}\right) \geq c_{1} \sin \left(\alpha_{3}+\alpha_{4}\right) \\
& w \leq c_{4} \sin \left(\alpha_{1}+\alpha_{2}\right) \geq u \sin \left(\alpha_{2}\right) \\
& w \leq c_{3} \sin \left(\alpha_{1}+\alpha_{2}\right) \geq u \sin \left(\alpha_{1}\right)
\end{array}
$$




$$
\begin{aligned}
& c_{1}+c_{2}+c_{3}+c_{4}=1 \\
& c_{4} \sin \left(\alpha_{1}\right)=c_{1} \sin \left(\alpha_{4}\right) \\
& c_{3} \sin \left(\alpha_{2}\right)=c_{2} \sin \left(\alpha_{3}\right) \\
& u=c_{4} \cos \left(\alpha_{1}\right)+c_{1} \cos \left(\alpha_{4}\right) \\
& u=c_{3} \cos \left(\alpha_{2}\right)+c_{2} \cos \left(\alpha_{3}\right) \\
& 0 \leq \alpha_{1} \leq \alpha_{2} \leq \pi \\
& 0 \leq u \leq \frac{1}{2} \\
& 0 \leq c_{i} \leq \frac{1}{2} \quad i=1,2,3,4 .
\end{aligned}
$$

Using an exact global optimization code $[15,16]$ based on Branch and Bound in continuous variables and interval analysis [17, 19], we proved in about 40 hours of computing time on a $\mathrm{PC}$ with $1.86 \mathrm{GHz}$ cycle time and $1 \mathrm{~GB}$ of memory that there is no solution with a value larger than that of Theorem 2 with an error less than $10^{-4}$.

Remark The quadrilateral with unit perimeter and maximum width of Theorem 2 is not homothetic to any member of the infinite set of quadrilaterals with unit diameter and maximum width [6].

\section{Bounds on the Width of Unit-Perimeter $n$-gons for $n=2^{s}$ with Integer $s \geq 3$}

For $n=2^{s}$ with integer $s \geq 3$, exact solutions, obtained either analytically or numerically, appear to be presently out of reach. However, approximate solutions, including near optimal ones, can be obtained in several ways.

The first way consists in perturbing the optimal solution of the problem with $n^{\prime}=$ $2^{s}-1$ vertices. To this effect, one may add a vertex in the middle of a side of the regular $n^{\prime}$-sided polygon $\mathcal{P}_{n^{\prime}}^{r}$. We denote the resulting $n$-gon by $R_{n}$. As next shown, this gives a solution better than the regular $n$-gon with unit perimeter $\mathcal{P}_{n}^{r}$.

Proposition 5 If $n=2^{s}$ for some integer $s \geq 3$, then

$$
w\left(R_{n}\right)=w\left(\mathcal{P}_{n^{\prime}}^{r}\right)=\frac{1}{2 n^{\prime}} \cot \left(\frac{\pi}{2 n^{\prime}}\right)>w\left(\mathcal{P}_{n}^{r}\right)=\frac{1}{n} \cot \left(\frac{\pi}{n}\right) .
$$

Proof Since the function $f(\alpha)=\alpha \cot (\alpha)$ is decreasing over the interval $] 0, \pi$, we have

$$
w\left(\mathcal{P}_{n^{\prime}}^{r}\right)=\frac{1}{\pi} f\left(\frac{\pi}{4 n-2}\right)>\frac{1}{\pi} f\left(\frac{\pi}{2 n}\right)=w\left(\mathcal{P}_{n}^{r}\right) .
$$

The widths of the regular polygon $\mathcal{P}_{n}^{r}$ and of the modified polygon $R_{n}$ (for $n=8$, 16, and 32) are given in Table 1 for comparison purposes. 
The second way consists in examining solutions for problems similar to the one studied in this paper. Mossinghoff [18] presents unit diameter polygons, denoted by $U_{n}$, with large perimeter. These polygons were obtained through local optimization assuming particular diameter configurations and an axis of symmetry. Mossinghoff analyzes the quality of these solutions by proving valid bounds on the errors. The diameter configurations of $U_{n}$ for $n=8,16$, and 32, are given on page 376 of [18] (the best diameter configuration and the corresponding polygon for $n=8$ was independently found and proved to be optimal in [2]). The widths of the corresponding polygons with unit perimeter, also denoted by $U_{n}$, are

$$
\begin{gathered}
w\left(U_{8}\right)=\frac{\cos \left(\frac{\alpha_{1}\left(U_{8}\right)}{2}\right)}{p\left(U_{8}\right)} \approx 0.3128368 \ldots, \\
w\left(U_{16}\right)=\frac{\cos \left(\frac{\alpha_{1}\left(U_{16}\right)}{2}\right)}{p\left(U_{16}\right)} \approx 0.3172088 \ldots, \\
w\left(U_{32}\right)=\frac{\cos \left(\frac{\alpha_{1}\left(U_{32}\right)}{2}\right)}{p\left(U_{32}\right)} \approx 0.3180495 \ldots,
\end{gathered}
$$

where the angles are $\alpha_{1}\left(U_{8}\right)=0.43528 \ldots, \alpha_{1}\left(U_{16}\right)=0.20123 \ldots, \alpha_{1}\left(U_{32}\right)=$ $0.098779 \ldots$, and the perimeters are $p\left(U_{8}\right)=3.1211471 \ldots, p\left(U_{16}\right)=3.1365544 \ldots$, and $p\left(U_{32}\right)=3.1403311 \ldots$, see Tables 5 and 7 in [18]. The values of the widths $w\left(U_{8}\right), w\left(U_{16}\right)$, and $w\left(U_{32}\right)$ are also reported in Table 1.

The third way is to apply global optimization to find polygons, with the same or other diameter configurations as mentioned above, that provide a solution with presumably maximum width and unit diameter. While a priori diameter configurations for polygons with maximum area, or with maximum perimeter, and unit diameter are known and are the same, i.e., connected caterpillars or odd cycles with pending edges, it is not known whether this still holds for maximum width polygons. Nevertheless, using them gives good results. For $n=8$, the diameter configuration 29 out of 31 possible ones (see Fig. 1 and pp. 48-49 of [5]) gives the best result [1]. The width of the corresponding polygon $U_{8}^{\prime}$ with unit diameter is approximately $0.3132296 \ldots$, see Table 1.

The fourth way is to provide general mathematical programs for maximizing the width of $n$-sided unit-perimeter polygons with $n=2^{s}$. We do this in two ways: first,

Table 1 Values of the widths of some approximate solutions

\begin{tabular}{llll}
\hline & $n=8$ & $n=16$ & $n=32$ \\
\hline$w\left(\mathcal{P}_{n}^{r}\right)$ & 0.3017767 & 0.3142087 & 0.3172866 \\
$w\left(R_{n}\right)=w\left(\mathcal{P}_{n^{\prime}}^{r}\right)$ & 0.3129490 & 0.3171455 & 0.3180374 \\
$w\left(U_{n}\right),[18]$ & 0.3128368 & 0.3172088 & 0.3180495 \\
$w\left(U_{n}^{\prime}\right),[1]$ & 0.3132296 & - & - \\
$w\left(U_{n}^{*}\right)$ & $\mathbf{0 . 3 1 4 1 1 4 4}$ & $\mathbf{0 . 3 1 7 2 8 4 9}$ & $\mathbf{0 . 3 1 8 0 5 4 2}$ \\
Upper bound & 0.3142087 & 0.3172866 & 0.3180542 \\
Gap & $<9.43 \times 10^{-5}$ & $<1.69 \times 10^{-6}$ & $<1.43 \times 10^{-8}$ \\
\hline
\end{tabular}


Fig. $6 n$-gon with $n=2^{s}$ with $s \geq 3$, general case

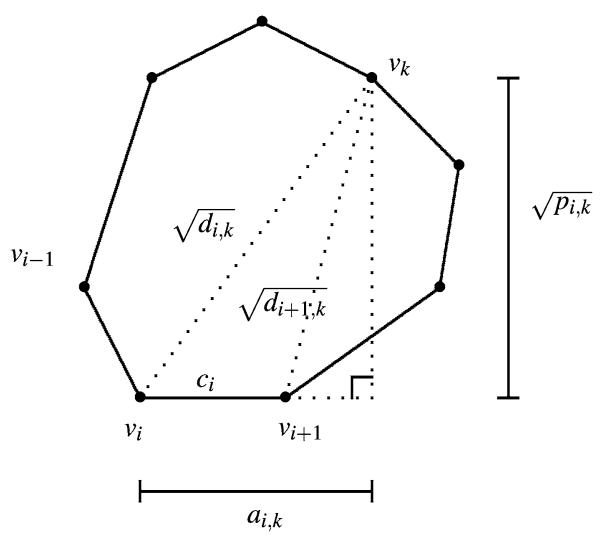

we propose a quadratic programming problem with a linear objective function and linear as well as quadratic nonconvex constraints; second, by eliminating some variables we obtain a more concise nonlinear and nonconvex program with nonconvex constraints involving higher-order terms as well as ratios.

In order to express the quadratic program we need further notation: For any $i$ and $j$ in $N=\{1,2, \ldots, n\}$ with $i<j$ and for any $(i, k)$ in $L=(i, k) \in\{N \times N: k \neq$ $i, k \neq i+1\}$, we introduce variables that represent:

$c_{i}$ : length of the side $\left[v_{i}, v_{i+1}\right]$,

$d_{i, j}: \quad$ square of the distance between the vertices $v_{i}$ and $v_{j}$,

$p_{i, k}$ : square of the distance from $v_{k}$ to the line containing $\left[v_{i}, v_{i+1}\right]$,

$a_{i, k}$ : positive length such that $a_{i, k}^{2}+p_{i, k} d_{i, k}$.

Theorem 3 The maximum width of a unit-perimeter convex $n$-gon is the square root of the optimal value of the program

$$
\begin{array}{ll} 
& \max _{c, d, p, a, z, \lambda, x, y} z \\
\text { s.t. } & z \leq \sum_{(i, k) \in L} \lambda_{i, k} p_{i, k} \quad \forall i \in N \\
& \sum_{(i, k) \in L} \lambda_{i, k}=1 \quad \forall i \in N \\
& \sum_{i \in N} c_{i}=1 \\
& d_{i, j}=\left(x_{i}-x_{j}\right)^{2}+\left(y_{i}-y_{j}\right)^{2} \quad \forall i \in N, j \in N, i<j \\
& c_{i}^{2}=d_{i, i+1} \quad \forall i \in N \\
& c_{i}^{2}-2 c_{i} a_{i, k}=d_{i+1, k}-d_{i, k} \quad \forall(i, k) \in L \\
& a_{i, k}^{2}+p_{i, k}=d_{i, k} \quad \forall(i, k) \in L \\
& a_{i, k} \geq 0 \quad \forall(i, k) \in L
\end{array}
$$




$$
\begin{aligned}
& p_{i, k} \geq 0 \quad \forall(i, k) \in L \\
& c_{i} \geq 0 \quad \forall i \in N \\
& \lambda_{i, k} \geq 0 \quad \forall(i, k) \in L .
\end{aligned}
$$

Without loss of generality, the following constraints can be added:

$$
c_{1} \geq c_{i} \quad \forall i \in N \backslash\{1\} \quad \text { and } \quad c_{2} \geq c_{n} .
$$

Proof The width $h_{i}$ associated with side $\left[v_{i}, v_{i+1}\right]$ is the square root of $\max \left\{p_{i, k}\right.$ : $(i, k) \in L\}$. This means that $h_{i}$ is the largest value for which there exists some scalars $\lambda_{i, k} \geq 0$ with $\sum_{(i, k) \in L} \lambda_{i, k}=1$ such that $h_{i}^{2} \leq \lambda_{i, k} p_{i, k}$ for every $k \in N$ with $k \neq i$ and $k \neq i+1$.

The width $w$ of the polygon is the least of these $n$ widths $h_{i}$, i.e., $w$ is the largest value such that $w \leq h_{i}$ for all $i \in N$. Therefore, the width of a polygon is the largest value of $w$ for which there exists some vector $\lambda$ in

$$
\begin{aligned}
& w^{2} \leq \lambda_{i, k} p_{i, k} \quad \forall(i, k) \in L \\
& \sum_{(i, k) \in L} \lambda_{i, k}=1 \quad \forall i \in N \\
& \lambda_{i, k} \geq 0 \quad \forall i \in N .
\end{aligned}
$$

The other constraints in the statement of the theorem simply ensure that the values $p_{i, k}$ represent the square of the distance from the vertex $v_{k}$ to the supporting line $L_{i}$ of the side $\left[v_{i}, v_{i+1}\right]$.

The additional constraints involving only the $c_{i}$ 's impose that $c_{1}$ is the longest side and $c_{2}$ is larger than $c_{n}$.

The nonconvex quadratic program (8) has $\frac{7}{2} n^{2}-\frac{7}{2} n+1$ variables and $\frac{5}{2} n^{2}-\frac{3}{2} n+$ 1 constraints. Eliminating all variables $a_{i, k}, d_{i, k}, p_{i, k}$, and $c_{i}$ yields a more concise nonlinear mathematical program

$$
\begin{array}{ll}
\max _{w, \lambda, x, y} & w^{2} \\
\text { s.t. } & w^{2} \leq \sum_{(i, k) \in L} \lambda_{i, k} \frac{\left(\left(y_{i+1}-y_{i}\right) x_{k}+\left(x_{i}-x_{i+1}\right) y_{k}+\left(x_{i+1} y_{i}-x_{i} y_{i+1}\right)\right)^{2}}{\left(x_{i+1}-x_{i}\right)^{2}+\left(y_{i+1}-y_{i}\right)^{2}} \\
& \forall i \in N \\
& \sum_{(i, k) \in L} \lambda_{i, k}=1 \quad \forall i \in N \\
& \sum_{i \in N} \sqrt{\left(x_{i+1}-x_{i}\right)^{2}+\left(y_{i+1}-y_{i}\right)^{2}}=1 \\
& \lambda_{i, k} \geq 0 \quad \forall(i, k) \in L .
\end{array}
$$

This program has only $n^{2}+1$ variables and $2 n+1$ constraints. 
Table 2 Vertices $(x, y)$ of $U_{n}^{*}$ with $x_{i} \geq 0$

\begin{tabular}{llll}
\hline$n$ & $(x, y)$ & & \\
\hline 8 & $(0,0.3229012)$ & $(0.09800651,0.2497652)$ & $(0.1604472,0.1532725)$ \\
& $(0.1350638,0.0317559)$ & $(0,0.00046964)$ & \\
16 & $(0,0.3189329)$ & $(0.05843830,0.3009091)$ & $(0.1124204,0.2722723)$ \\
& $(0.1414934,0.2173247)$ & $(0.1594171,0.1582299)$ & $(0.1532569,0.09596931)$ \\
& $(0.1128727,0.04654642)$ & $(0.06374517,0.00656046)$ & $(0.06374517,0.00656046)$ \\
& $(0,-0.00005686)$ & & \\
& $(0,0.3184416)$ & $(0.03072433,0.3138643)$ & $(0.06085961,0.3063348)$ \\
& $(0.08752067,0.2903341)$ & $(0.1125001,0.2718262)$ & $(0.1310448,0.2467992)$ \\
& $(0.1470609,0.2200932)$ & $(0.1546403,0.1898071)$ & $(0.1592192,0.1589349)$ \\
& $(0.1576845,0.1277197)$ & $(0.1471372,0.09822279)$ & $(0.1337373,0.06991056)$ \\
& $(0.1126671,0.0466453)$ & $(0.0894032,0.02557878)$ & $(0.06099882,0.01212665)$ \\
& $(0.03140340,0.0015557)$ & $(0,-0.00000522)$ & \\
\hline
\end{tabular}

Local optimal solutions of the program (9) were obtained for $n=8,16$, and 32 using the procedure fmincon of MatLab ${ }^{\mathrm{TM}}$. These solutions are denoted by $U_{n}^{*}$. Computing times on a $1.6 \mathrm{GHz} \mathrm{PC}$ with $512 \mathrm{MB}$ were of about 1 second, 20 seconds, and 20 minutes respectively. Best values found for the widths are given in Table 1 and vertex coordinates for the corresponding $n$-gons in Table 2 . As the best solutions found were symmetric with respect to a vertical diagonal, only points with $x_{i} \geq 0$ are described. Observe the small values of the errors and their rapid decrease with increase of $n$. Note that starting points were the $n$-gons called $U_{n}$ in [18]. Other starting points, e.g., randomly generated ones, did not lead to improved solutions. Values for all approximate solutions for $n=8,16$, and 32 are presented in Table 1, the best values being in bold.

\section{References}

1. Audet, C., Hansen, P., Messine, F.: Quatre petits octogones. Matapli 80, 39-59 (2006)

2. Audet, C., Hansen, P., Messine, F.: The small octagon with longest perimeter. J. Comb. Theory Ser. A 114, 135-150 (2007)

3. Audet, C., Hansen, P., Messine, F.: Extremal problems for convex polygons. J. Glob. Optim. 38, 163179 (2007)

4. Audet, C., Hansen, P., Messine, F., Perron, S.: The minimum diameter octagon with unit-length sides: Vincze's wife's octagon is suboptimal. J. Comb. Theory Ser. A 108, 63-75 (2004)

5. Audet, C., Hansen, P., Messine, F., Xiong, J.: The largest small octagon. J. Comb. Theory Ser. A 98(1), 46-59 (2002)

6. Bezdek, A., Fodor, F.: On convex polygons of maximal width. Arch. Math. 74(1), 75-80 (2000)

7. Blåsjö, V.: The isoperimetric problem. Am. Math. Mon. 112, 526-566 (2005)

8. Brass, P., Moser, W., Pach, J.: Research Problems in Discrete Geometry. Springer, New-York (2005)

9. Croft, H.T., Falconer, K.J., Guy, R.K.: Unsolved Problems in Geometry. Springer, New York (1991)

10. Datta, B.: A discrete isoperimetric problem. Geom. Dedic. 64, 55-68 (1997)

11. Heath, G.H.: A History of Greek Mathematics, vol. 2. Clarendon Press, Oxford (1921)

12. Larman, D.G., Tamvakis, N.K.: The decomposition of the $n$-sphere and the boundaries of plane convex domains. In: Convexity and Graph Theory (Jerusalem, 1981), vol. 87, pp. 209-214 (1984) 
13. Lassak, M.: Reduced convex bodies in the plane. Isr. J. Math. 70, 365-379 (1990)

14. Lassak, M., Gritzmann, P.: Estimates for the minimal width of polytopes inscribed in convex bodies. Discrete Comput. Geom. 4, 627-635 (1989)

15. Messine, F.: Deterministic global optimization using interval constraint propagation techniques. RAIRO. Oper. Res. 38(4), 277-294 (2004)

16. Messine, F.: A deterministic global optimization algorithm for design problems. In: Audet, C., Hansen, P., Savard, G. (eds.) Essays and Surveys in Global Optimization, pp. 267-294. Springer, Berlin (2005)

17. Moore, R.E.: Interval Analysis. Prentice Hall, Englewood Cliffs (1966)

18. Mossinghoff, M.J.: Isodiametric problems for polygons. Discrete Comput. Geom. 36, 363-379 (2006)

19. Ratschek, H., Rokne, J.: New Computer Methods for Global Optimization. Ellis Horwood, Chichester (1988)

20. Reinhardt, K.: Extremale polygone gegebenen durchmessers. Jahresber. Deutsch. Math. Verein 31, 251-270 (1922)

21. Reuleaux, F.: The Kinematics of Machinery. Dover, New York (1963). Translation of German original

22. Tamvakis, N.K.: On the perimeter and the area of the convex polygon of a given diameter. Bull. Greek Math. Soc. 28, 115-132 (1987)

23. Thomas, I.: Illustrating the History of Greek Mathematics, vol. 2. Harvard Univ. Press, Cambridge (1941)

24. Vincze, S.: On a geometrical extremum problem. Acta Sci. Math. (Szeged) 12, 136-142 (1950) 